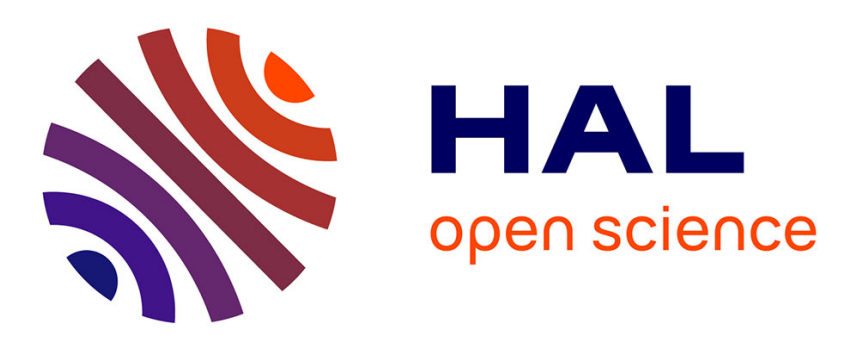

\title{
Iris Identification Based on a Local Analysis of the Iris Texture
}

\author{
Mathieu Adam, Florence Rossant, Beata Mikovicova, Frédéric Amiel
}

\section{To cite this version:}

Mathieu Adam, Florence Rossant, Beata Mikovicova, Frédéric Amiel. Iris Identification Based on a Local Analysis of the Iris Texture. ISPA, Sep 2009, Salzburg, Austria. 10.1109/ISPA.2009.5297683 . hal-02882942

\section{HAL Id: hal-02882942 \\ https://hal.science/hal-02882942}

Submitted on 28 Jun 2020

HAL is a multi-disciplinary open access archive for the deposit and dissemination of scientific research documents, whether they are published or not. The documents may come from teaching and research institutions in France or abroad, or from public or private research centers.
L'archive ouverte pluridisciplinaire HAL, est destinée au dépôt et à la diffusion de documents scientifiques de niveau recherche, publiés ou non, émanant des établissements d'enseignement et de recherche français ou étrangers, des laboratoires publics ou privés. 


\title{
Iris Identification Based on a Local Analysis of the Iris Texture
}

\author{
Mathieu Adam, Florence Rossant, Beata Mikovicova, Frédéric Amiel \\ ISEP (Institut Supérieur d'Electronique de Paris), SI2A Laboratory \\ 21 rue d'Assas, 75006 Paris, France \\ mathieu.adam|florence.rossant|beata.mikovicova|frederic.amiel@isep.fr
}

\begin{abstract}
This paper focuses on a new iris identification method based on a local analysis of the iris texture. In the method, the iris is divided in sub-regions, using locally sliding windows, to extract local signatures. Local distances are then calculated and fused, based on a weighting average. The sliding allows to compensate for local distortions due to segmentation imprecision. The applied weights take into account additional knowledge about the information quantity carried by the different sub-regions and its reliability.

Tests have been conducted on the CASIA-IrisV3Interval database. They show good performances of the new method. Similar or even better results are obtained, compared to published ones, with a set of iris containing twice more subjects.
\end{abstract}

\section{Introduction}

Identification systems using iris biometrics have been widely studied since the iris is characterized by a random morphogenesis and stability over time. It is worldwide considered as the most reliable biometrics. Indeed, these systems, in comparison with systems using other modalities, perform the highest recognition rates.

Iris identification systems proposed so far present the following methodology [1-7]: the iris is segmented, taking possibly into account occlusions by eyelids $[4,5]$, a signature is then extracted from the iris texture and finally a distance measure is used to compare the tested signature with reference signatures stored in a database. These systems often process iris images of poor quality. Images are blurred, defocused or occluded by eyelids, eyelashes or light reflections, because of acquisition difficulties. Most of the published studies focus on improving the identification system robustness in order to deal with these degradations. Different approaches have been proposed: multiple signatures are used [1,2], the coefficients of the signature, corresponding to areas that are likely to be occluded, are systematically excluded from the signature $[3,6,7]$, the pertinent information is identified dynamically to improve the distance measure [4,5].

This article presents improvements of our iris recognition system [1][8]. This system relies classically on three main steps: iris segmentation and unwrapping, signature computation, signature comparison. The signatures are calculated from an orthogonal wavelet packet decomposition, leading to a robust identification method with respect to contrast variations or blurring, as long as the segmentation is accurate [1]. Then we developed an eyelid localization algorithm [8], which allows to restrict the signature comparison on non occluded coefficients only, similarly to [4]. In this paper, we propose a new signature comparison method based on a fusion of local distances. The purpose is to take into account local distortions in the unwrapped image that are due to segmentation imprecision. Another idea is to give more importance to iris areas which are likely to provide more reliable information.

The paper is organized as follows. Section 2 discusses the global distance measure and its limitation. Sections 3 and 4 focus on the local distance measures and the applied fusion rule. Finally, tests and results are presented in Section 5.

\section{Identification algorithm}

We present in this section the distance measure used for comparing two iris signatures. We also discuss the limitations of the method with regard to segmentation imprecision.

\subsection{Distance measure}

We proposed an iris comparison based on a distance measure combining signatures extracted from two wavelet packets (denoted by P2 and P10) at the third level of decomposition [1]. Each packet is coded on 3 levels $(-1,0,+1)$ to provide the signature. Now, we introduce the eyelid segmentation results in the iris comparison step. Let $S_{i}^{R}$ and $S_{i}^{T}$ be respectively the reference and the tested signatures extracted from the wavelet packet $P i$, and $M^{R}$ and $M^{T}$ be the binary masks defining the non occluded coefficients corresponding to iris texture [8]. The distance measure is defined by:

$$
\begin{aligned}
& H D_{i}=\frac{1}{2 N_{i r i s}} \sum_{j, k}\left|S_{i}^{T}(j, k)-S_{i}^{R}(j, k)\right| \cdot M^{T}(j, k) \cdot M^{R}(j, k)(1) \\
& D=2 \cdot H D_{2} H D_{10}
\end{aligned}
$$

where $\mathrm{N}_{\text {iris }}$ is the number of non occluded coefficients:

$$
N_{i r i s}=\sum_{j, k} M^{T}(j, k) \cdot M^{R}(j, k)
$$


In this way, the signatures are compared on their common non-occluded part corresponding to iris texture only.

In our system, two signatures are stored in the reference database for each individual. The tested signature is compared to both signatures of each individual and the minimum distance is retained to provide the final measure of dissimilarity.

\subsection{Limitations related to the use of a global measure}

In our system, the iris is extracted by maximizing the mean gradient along the pupil and the external iris boundaries, which are respectively modeled by a circle and an ellipse [1]. Many parameters related to the image acquisition process may impact the results. The optical axis, the illumination conditions, the pupil dilatation, the partial occlusions by eyelids or eyelashes are the main causes of segmentation variability: displacement of the circle or ellipse center, change of the radius or ellipse axis parameters. This variability results in translations of the iris structures in the unwrapped image, as well as dilatation or contraction, especially in the radial direction.

A global distance measure cannot deal with such local distortions. Thus we propose to combine local distances made on eight sliding windows. The shifts of the windows are mutually independent. Thus, it is possible to better compensate for local distortions that are not uniform over the unwrapped image. Moreover, this cutout allows to refine the analysis by weighting the local distances according to the reliability of the information carried by each block.

\section{Local analysis method and fusion}

\subsection{Principle}

The iris is divided in eight areas, equivalent to eight rectangular sub-windows in the unwrapped image, as illustrated in Figure 1.

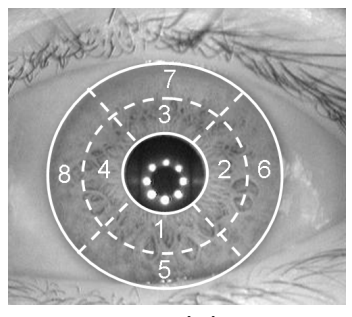

(a)

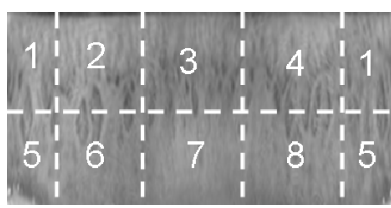

(b)
Figure. 1. The 8 areas in the source image (a) corresponding to 8 rectangular blocks in the unwrapped image (b) (128x256)

Blocks 1 to 4 correspond to the texture close to the pupil. As blocks 5 to 7 are related to peripheral textures, they are more prone to occlusions, especially blocks 7 and 5 .
Figure 2 shows the functional scheme of the proposed identification system. The first step consists in a global angular shifting to compensate for eye rotation between the reference and the tested image [1].

Let us denote by $N$ the maximum shift, in pixels, of the sub-windows. The tested unwrapped image is extended by $N$ pixels in both directions to authorize horizontal and vertical sliding of the sub-windows with minimized side effects.

The texture comparison is then realized on each rectangular block of the unwrapped image independently on the others. The blocks of the tested unwrapped image slide along the vertical and horizontal directions, around their central position, while the subwindows of the reference image are fixed. A subsignature is extracted from each block for the $(2 N+1)(2 N+1)$ possible translations and compared to the corresponding reference sub-signature, according to equation (1). This process leads to a set of $(2 N+1)(2 N+1)$ distance measures for each block, which are then fused to provide the final distance.

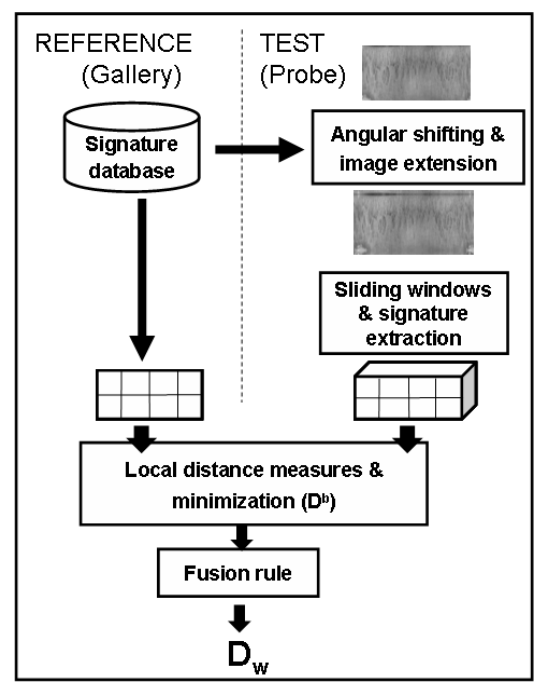

Figure 2. Functional scheme of the identification method

\subsection{Local distances and minimization}

Let us denote by $S_{i,\{m, n\}}^{T, b}(j, k)$ the coefficients of the signature derived from packet $P i$, for the sub-window $b$ ( $b \in[1,8]$ ) of the tested iris $T$ and the translation $\{m, n\}$. We denote by $S_{i}^{R, b}(j, k)$ its equivalent for the reference iris $R$. The distances $D_{\{m, n\}}^{b}$ between the signatures are computed according to equation (1) for each translation $\{m, n\}$. The optimal superposition between the tested and the reference sub-signatures is given by the minimum distance $D^{b}$ :

$$
D^{b}=\min _{m, n} D_{\{m, n\}}^{b}, b \in[1,8]
$$

Thus, eight distances are obtained, each one corresponding to the analysis of a sub-window. The 
next step aims at merging these local distances to get a final measure representing the global dissimilarity of both iris.

\subsection{Fusion of the local distances}

Different fusion rules can be applied, such as the minimum or maximum, the arithmetical or geometrical average [9].

In our application, we take into account some additional knowledge. The general idea is to give more importance to the local distances corresponding to the most informative and reliable areas. Therefore, we choose a weighting sum as fusion rule. It allows to combine all pieces of information.

We define two weights: the first one, denoted by $w_{d}^{b}$, represents the proportion of coefficients corresponding to non occluded iris texture and is set dynamically.

The purpose of the second weight, $w_{s}^{b}$, is to limit the influence of blocks that are likely to be unreliable or non discriminating. Indeed, we expect all the local distances to be similar, high (near 0.4) when comparing iris images of different subjects and low (near 0.1) when comparing iris images of the same subject. In the first case, a local distance may be lower than the others when the considered block is not enough discriminating (comparison of low textured iris areas for example) or improperly shifted (false matching decreasing the distance). In the second case, a distance measure can be higher than the others when distortions are not properly compensated (translations only are considered) or when defects (eyelashes, light spots, etc.) noise the measure. Consequently, we propose to give less importance to the local measures that are statistically different from the others. For that, a second weight is dynamically defined and applied as follows.

Let $m$ be the mean and $\sigma$ the standard deviation of the eight local distances $D^{b}$ obtained between the tested iris and a reference iris. The weight, denoted as $w_{s}^{b}$ is given by the following equation:

$$
w_{s}^{b}=\left\{\begin{array}{l}
p \quad \text { if }\left|D^{b}-m\right|>\sigma \\
1 \quad \text { otherwise }
\end{array}\right.
$$

where $p$ is less than 1. This parameter is learnt from a training set as the value minimizing the error rates.

The final weight of block $b$ is defined by:

$$
w^{b}=\frac{w_{d}^{b} * w_{s}^{b}}{\sum_{k=1}^{8} w_{d}^{k} * w_{s}^{k}}
$$

Finally, the global distance between the tested and the reference iris is given by:

$$
D_{w}=\sum_{b=1}^{8} w^{b} * D^{b}
$$

\section{Parameter training}

This new identification algorithm requires two parameters: the maximum shift $N$ and the parameter $p$ of the weight $w_{s}^{b}$. Both are learnt from a subset of the iris database.

\subsection{Iris database and training method}

The CASIA-IrisV3-Interval database [10] is used in all experiments. We extracted a sub-database including 888 images of 222 subjects. There are two images per subject both in the probe (i.e. test) set and the gallery (i.e. reference) set.

The parameter training is realized by optimizing the performances of the identification system. For that, several error rates are computed: the false accept rate (FAR) measures the rate of impostors accepted by the system, the false reject rate (FRR) measures the rate of authentics rejected by the system, the equal error rate (EER) is the rate where FAR=FRR. Moreover, we study the error rate in high security mode: the FRR when the FAR is zero (FRR(FAR $=0 \%)$ ).

A training set is extracted from the CASIA subdatabase. It contains the first half of subjects (Fig.3): 111 subjects and 444 images. The training consists in selecting the parameters that minimize the error rates defined above. This set of parameters is then validated on a test set, also extracted form the CASIA subdatabase. The test set contains the second half of subjects, so again 111 subjects and 444 images. It is worth noting that the training set and the test set are completely independent. In particular, the tested iris of

\begin{tabular}{|c|c|c|c|c|c|c|c|c|}
\hline \multicolumn{2}{|c|}{ GALLERY } & \multicolumn{3}{|c|}{ Training set } & \multicolumn{2}{|c|}{ PROBE } & & \\
\hline$l: l$ & $1: 2$ & $1: 3$ & $1: 4$ & $\ldots$ & $111: 4$ & $112: 3$ & $\ldots 222: 3$ & $222: 4$ \\
\hline $2: 1$ & $2: 2$ & 1.3 & 2.4 & $\ldots$ & $111: 4$ & $112: 3$ & $\ldots 222: 3$ & $222: 4$ \\
\hline$\cdots$ & $\cdots$ & $\cdots$ & $\cdots$ & $\cdots$ & $\cdots$ & $\cdots$ & $\cdot \cdot$ & $\cdots$ \\
\hline $111: 1$ & $111: 2$ & 1.3 & 2.4 & $\ldots$ & $111: 4$ & $112: 3$ & $\ldots 222: 3$ & $222: 4$ \\
\hline $112: 1$ & $112: 2$ & 1.3 & 2.4 & $\ldots$ & $111: 4$ & $112: 3$ & $\ldots 222: 3$ & $222: 4$ \\
\hline$\ldots$ & $\ldots$ & $\cdots$ & $\cdots$ & & $\cdots$ & $\cdots$ & .. & $\cdots$ \\
\hline $221: 1$ & $222: 2$ & 1.3 & 2.4 & $\ldots$ & $111: 4$ & $112: 3$ & $\ldots 222: 3$ & $222: 4$ \\
\hline $222: 1$ & $222: 2$ & 2.3 & 2.4 & $\ldots$ & $111: 4$ & $112: 3$ & $\ldots 222: 3$ & $222: 4$ \\
\hline
\end{tabular}
the training set are not compared with the reference iris of the test set, and conversely (Fig.3).

Test set

Figure 3. Extraction of the learning set and the test set from the CASIA sub-database.

Each image is referenced by 2 numbers: Identity Descriptor : Image Descriptor

\subsection{Maximum shifting parameter $N$}

The first parameter to be set is $N$, the maximum shifting of the sub-windows. It has been evaluated by computing the local distances $D^{b}$, which are then fused using the weighted average $((3)(4)(5))$ with $p=1$. Thus, 
only the weight $w_{d}^{b}$ is considered. Table 1 shows the results for different values of $N$.

Table 1. Evaluation and validation of the shifting parameter $N$

\begin{tabular}{|c|c|c|c|c|c|}
\hline \multicolumn{2}{|l|}{} & \multicolumn{2}{|c|}{ Training set } & \multicolumn{2}{c|}{ Test set } \\
\hline $\mathrm{N}$ & Shifts & EER & FRR(FAR=0\%) & EER & FRR(FAR=0\%) \\
\hline 0 & 0 & $2.25 \%$ & $8.11 \%$ & $2.70 \%$ & $6.76 \%$ \\
\hline 1 & 9 & $1.35 \%$ & $6.76 \%$ & $2.25 \%$ & $4.95 \%$ \\
\hline 2 & 25 & $1.35 \%$ & $4.50 \%$ & $1.80 \%$ & $4.05 \%$ \\
\hline 3 & 49 & $1.80 \%$ & $3.15 \%$ & $1.80 \%$ & $3.60 \%$ \\
\hline 4 & 81 & $1.35 \%$ & $4.05 \%$ & $1.80 \%$ & $3.15 \%$ \\
\hline
\end{tabular}

The best performances are obtained on the training set for $N=3$ and $N=4$. The $\mathrm{FRR}(\mathrm{FAR}=0 \%)$ is slightly better on the test set for $N=4$. However, setting $N=4$ instead of $N=3$ increases significantly the computing complexity: 81 shifts against 49 . For this reason, the learning of the parameter $p$ will be done with both values (Section 4.3). All these results also confirm the necessity of shifting the sub-windows to deal with local distortions. Moreover, it is worth noting that the optimal values are consistent with the translations we can generally observe by directly comparing unwrapped images coming from the same iris.

\subsection{Weighting parameter $p$}

The second parameter to be set is the value $p$ used in the definition of the weight $w_{s}(3)$. For the training, we use $N=3$ or $N=4$, and we apply the weighting-sum fusion rule $((3)(4)(5))$.

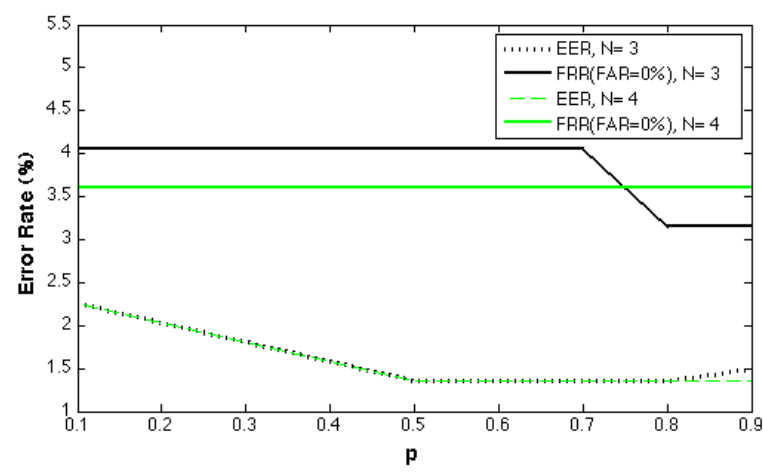

Figure 4. Evaluation of the parameter $p$ on the training set

Figure 4 shows the EER and the FRR for a zero FAR, obtained on the training set. These error rates are minimized for $N=3$ and $p=0.8$, and for $N=4$ and $p \approx 0.7$. The performances on the training set are slightly better in the first case. The performances on the test set are similar, validating the learnt values: $E E R=1.35 \%$ and $F R R(F A R=0 \%)=3.15 \%$ in both cases. So we retain the configuration leading to the lowest complexity: $N=3$, $p=0.8$.

\section{Tests and results}

The proposed method has been then evaluated on the entire sub-database extracted from the CASIA-IrisV3Interval database [10], containing 888 images of 222 subjects.

\subsection{Validation of the global distance measure}

The first set of experiments aims at validating the distance defined in equation (1), applied on the whole signature (no local measure). The whole database has been manually segmented. The performances are evaluated in 3 cases: in the first two cases, the occlusions are not taken into account (all mask coefficients are set to one), and the distance is computed from the signature using P2 only or P2 and $\mathrm{P} 10$, following respectively equations (6) and (7).

$$
\begin{aligned}
& D=\frac{1}{2 H W} \sum_{j, k}\left|S_{2}^{T}(j, k)-S_{2}^{R}(j, k)\right| \\
& D_{i}=\frac{1}{2 H W} \sum_{j, k}\left|S_{i}^{T}(j, k)-S_{i}^{R}(j, k)\right| \\
& D=2 . H D_{2} H D_{10}
\end{aligned}
$$

where $H W$ is the size of the signature $S_{i}$. In the third case, the occlusions are considered as defined in equation (1).

A decision-making power metric $d_{p}(8)$ is used in addition to the traditional error rates to measure the performances. It reflects the intrinsic separability of the authentic and impostor distributions [11].

$$
d_{p}=\frac{\left|\mu_{A}-\mu_{I}\right|}{\sqrt{\frac{1}{2}\left(\sigma_{A}^{2}+\sigma_{I}^{2}\right)}}
$$

where $\left(\mu_{\mathrm{A}}, \sigma_{\mathrm{A}}\right)$ and $\left(\mu_{\mathrm{I}}, \sigma_{\mathrm{I}}\right)$ are respectively the mean and the standard deviation of the authentic and impostor distributions.

Table 2. Performances evaluated on the manually segmented iris database

\begin{tabular}{|c|c|c|c|}
\hline & $d_{p}$ & EER & FRR(FAR=0\%) \\
\hline P2 & 5.24 & $0.06 \%$ & $1.80 \%$ \\
\hline P2P10 & 6.80 & $0.02 \%$ & $0.45 \%$ \\
\hline P2P10+Occlusion & 6.98 & $0.00 \%$ & $0.00 \%$ \\
\hline
\end{tabular}

The results (Tab. 2) confirm that the fusion of the signatures extracted from wavelet packets 2 and 10 increases the separability of the authentic and impostor distributions and decreases the error rates. Excluding the occluded coefficients still improve the performances. In this case, we have an identification system without errors, since the separation between the 
authentic and impostor distributions is positive. These performances, obtained with manually segmented images, will serve as benchmark in the following experiments.

\subsection{Evaluation of the measure based on the fusion of local distances}

In the second set of experiments, we introduce the automatic segmentation of the iris and the eyelids. Three different cases are considered (Tab. 3, Fig. 5). First, the distance is measured from $S_{2}$ and $S_{10}$ according to equation (7), i.e. without rejecting the occluded coefficients. Second, the occlusions are taken into account according to equation (1). Third, we introduce the local analysis and the fusion of local measures described in Section 3 (equation (5)). In this case, we use the optimal parameters according to the training results: $N=3$ pixels, $p=0.8$. Figure 5 presents the corresponding ROC curves, giving the FRR as a function of the FAR.

We observe again a significant improvement obtained with the consideration of the occlusions (Tab. 3 ), confirming the need of an automatic eyelid segmentation algorithm. The results are quite stable compared to the ones presented in the first test, thus validating our segmentation algorithms, for both iris boundary and eyelid detection. However the performances are slightly degraded: the EER is now equal to $0.90 \%$ instead of $0 \%$, and the $\mathrm{FRR}(\mathrm{FAR}=0 \%$ ) $=5.41 \%$ instead of $0 \%$. This loss proves the importance of an accurate segmentation.

The best performances are obtained with the fusion of the local distances. Compared to the global distance, the EER remains equal to $0.9 \%$ and the $\mathrm{FRR}(\mathrm{FAR}=0 \%$ ) decreases from $5.41 \%$ to $2.70 \%$, leading to a better reliability in high security mode.

Table 3. Performances evaluated on the automatically segmented iris database

\begin{tabular}{|c|c|c|}
\hline & EER & FRR(FAR=0\%) \\
\hline P2P10 & $1.35 \%$ & $7.66 \%$ \\
\hline P2P10+Occlusion & $0.90 \%$ & $5.41 \%$ \\
\hline Local Analysis & $0.90 \%$ & $2.70 \%$ \\
\hline
\end{tabular}

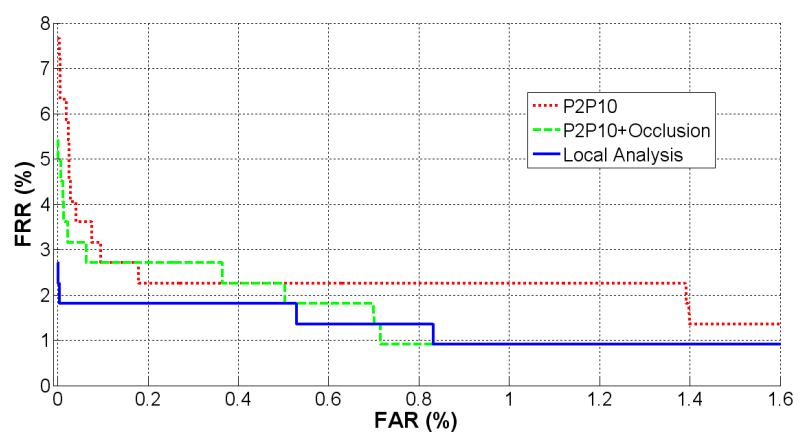

Figure 5. ROC curve comparison with automatic segmentation

\subsection{Evaluation of the fusion rules}

In this set of experiments, we evaluate the contribution of the defined weights. Table 4 presents the performances obtained with four different fusion rules: a simple average without any weighting (SA), the weight $w_{s}$ only, the weight $w_{d}$ only, and the combination of both weights $w_{s}$ and $w_{d}$.

Compared to the simple-average fusion rule (SA), the weighted averages $w_{s}$ and $w_{d}$ allow each to reach a better reliability, demonstrating that these fusion parameters are relevant. The gain obtained by weighting the occluded blocks is particularly significant. Combining then $w_{d}$ with the second weight $w_{s}$ conducts to the best performances, especially in the area of high security.

Table 4. Performances of the fusion rules

\begin{tabular}{|c|c|c|c|}
\hline \multicolumn{2}{|c|}{} & EER & FRR(FAR=0\%) \\
\hline \multirow{3}{*}{$\begin{array}{c}\text { Fusion } \\
\text { Rules }\end{array}$} & SA & $2.25 \%$ & $6.31 \%$ \\
\cline { 2 - 4 } & $w_{s}$ & $1.80 \%$ & $5.86 \%$ \\
\cline { 2 - 4 } & $w_{d}$ & $0.90 \%$ & $3.15 \%$ \\
\cline { 2 - 4 } & $w_{s} \& w_{d}$ & $0.90 \%$ & $2.70 \%$ \\
\hline
\end{tabular}

\subsection{Comparison with other iris identification systems}

The performances have also been compared to the ones published in the literature [2][5] (Tab. 5). The results presented by Daugman and Proença [2] were obtained on the CASIA database [10] with a subset of 800 images and 80 subjects, and by Chen [5] with a subset of 756 images and 108 subjects. We obtained similar or even better results for approximately the same number of iris images (888). However our database contains two or three more subjects (222) and so, these results demonstrate a very good robustness with respect to the increase of subjects.

Table 5. Performances reached by our local analysis method, compared with the literature

\begin{tabular}{|c|c|c|}
\hline & EER & FRR(FAR=0\%) \\
\hline Daugman [2] & $1.44 \%$ & $3.41 \%$ \\
\hline Proença [2] & $1.01 \%$ & $2.39 \%$ \\
\hline Chen [5] & $0.79 \%$ & Non evaluated \\
\hline Proposed & $0.90 \%$ & $2.70 \%$ \\
\hline
\end{tabular}

\section{Conclusion}

In this paper, a new identification method based on the local analysis of the iris texture has been proposed. This method allows to compensate for local distortions due to segmentation imprecision. To this end, local sliding sub-windows are used to perform local comparison between iris signatures. The resulting local distances are then fused to generate a global distance, based on a weighted average. This fusion rule takes into 
account the proportion of non occluded coefficients as well as the statistical distribution of the local distances.

Experiments have been performed on the CASIAIrisV3-Interval database to validate the proposed method. We first confirmed the interest of combining signatures extracted from two wavelet packets. We also showed the need of an accurate segmentation of the iris, including eyelid detection, and demonstrated the reliability of our segmentation algorithms. Finally, we evaluated the new distance measure based on the fusion of local distances and compared our system performances with the ones published up to now. We now obtain similar results on a set of iris images containing twice more subjects.

\section{References}

[1] F.Rossant, M.Torres Eslava, T.Ea, F.Amiel and A.Amara, "Iris identification and robustness evaluation of a wavelet packets based algorithm" International Conference in Image Processing, IEEE vol.3, Genova, 2005, pp. 257-260.

[2] H.Proença and L.A.Alexandre, "Toward noncooperative iris recognition: A classification approach using multiple signatures" Pattern Analysis and Machine Intelligence, IEEE vol.29, 2007, no. 4.

[3] C.Tisse, L.Martin, L.Torres and M.Robert, "Person identification technique using human iris recognition" International Conference on Vision Interface, IEEE vol.29, Canada, 2002, no. 4.
[4] J.Daugman, "New methods in iris recognition" Trans. Systems, Man, and Cybernetics, IEEE vol.37, 2007, no. 5.

[5] Y.Chen, S.C.Dass and A.K.Jain, "Localized iris image quality using 2-D wavelets" International Conference In Biometrics, IEEE vol.29, Hong Kong, 2006, no. 4.

[6] Z.Ma, M.Qi, H.Kang, S.Wang and J.Kong, "Iris Verification using wavelet moments and neural network" Life System Modeling and Simulation, vol.4689, Shanghai, 2007, pp. 218-226.

[7] J.Lee, P.S.Huang, C. Chang and T.Tu, "A novel approach for iris recognition using local edge patterns" Advances in Visual Computing, vol.4842, USA, 2007, pp. 479-488.

[8] M.Adam, F.Rossant, F.Amiel, B.Mikovikova and T.Ea, "Reliable Eyelid Localization for Iris Recognition" Advances Concepts for Intelligent Vision Systems, vol.5259, France, 2008, pp. 1062-1070.

[9] R.Snelick, U.Uludag, A.Mink, M.Indovina and A.K.Jain, "Large-Scale Evaluation of multimodal Biometric Authentication Using State-of-the-Art Systems" Pattern Analysis and Machine Intelligence, IEEE vol.27, 2005, no.3.

[10] CASIA Iris Image Database, Inst. Of Automation, Chinese Academy of Sciences, 2004

[11] J.Daugman, "Biometric Decision Landscapes" Technical Report no 482, Computer Laboratory, University of Cambridge, 2000 